\title{
Choice boosts curiosity
}

Patricia Romero Verdugo ${ }^{1,2 *}$, Lieke L. F. van Lieshout ${ }^{1,2}$, Floris P. de Lange ${ }^{1}$, \& Roshan Cools ${ }^{1,2}$

Affiliations:

${ }^{1}$ Donders Institute for Brain, Cognition and Behaviour, Radboud University, P.O. Box 9101, 6500 HB Nijmegen, The Netherlands.

${ }^{2}$ Department of Psychiatry, Radboud University Medical Centre, P.O. Box 9101, 6500 HB Nijmegen, The Netherlands.

Corresponding author $(*)$ :

Patricia Romero Verdugo

Donders Institute for Brain, Cognition and Behaviour, Radboud University

P.O. Box 9101

6500 HB Nijmegen

The Netherlands

E-mail: p.romeroverdugo@donders.ru.nl 


\begin{abstract}
In our connected era, we spend significant time and effort pursuing our curiosity. Often, we choose which information we seek, but on occasion, the selection is made for us. Hence, understanding whether and how choice influences curiosity is a key question. Prior evidence indicates that humans exhibit a preference for options they have freely chosen over equally valued options that they have not. Based on these findings, we hypothesized that we also exhibit enhanced curiosity in the context of choice compared with that of no-choice. To test this hypothesis, we designed a task in which participants saw two lotteries on each trial. On some trials, participants chose which lottery would be played. On other trials, the lottery was selected for them. Participants then indicated their curiosity about the outcome of the lottery that would be played in terms of self-report ratings (Experiment 1) or willingness to wait decisions (Experiment 2). As predicted, we found that participants exhibited higher curiosity ratings and higher willingness to wait for the outcome of lotteries they had chosen than for lotteries that had been selected for them (controlling for initial preference). These findings demonstrate that choice boosts curiosity, which may have implications for how to boost learning, memory, and motivation, for example in educational settings.
\end{abstract}

Keywords: curiosity; choice; uncertainty; expected value; information seeking 


\section{Introduction}

Curiosity, our internal drive for information, is pervasive in everyday life and is a key driver of behaviour (Kidd \& Hayden, 2015). Humans have a preference for information, and are willing to pay for it, even when it does not serve an immediate purpose or help us achieve an end goal. For instance, human participants were willing to give up monetary compensation to receive knowledge about lottery outcomes even though this knowledge did not help them perform better or maximise rewards (Charpentier et al., 2018). Non-human primates have also been shown to give up extrinsic rewards to receive advance information about upcoming rewards (Blanchard et al., 2015). This and other recent evidence (Bennett et al., 2016; Kang et al., 2009; Rodriguez Cabrero et al., 2019; van Lieshout et al., 2018) show that we assign intrinsic value to information: we seek it at the cost of other (extrinsic) rewards, even when it has no instrumental utility (Sharot \& Sunstein, 2020).

In our connected era, we have a practically infinite amount of information at our fingertips and devote extensive time seeking it to satiate our curiosity. However, our time and (cognitive) resources are limited, and hence information selection is inevitable. In some cases, we get to choose what information to consume (e.g., choosing what websites to browse), but in other cases (e.g., the traditional classroom), the selection is made for us. Given the pervasiveness of information seeking and the need for selection, understanding how choice of information sampling influences curiosity is key.

Extensive evidence from behavioural and brain imaging studies indicates that the act of choosing an option increases its subjective value (Ariely \& Norton, 2008; Brehm, 1956; Izuma et al., 2010; Sharot et al., 2009, 2010). It is less clear however whether choosing which information to sample also increases the subjective value of information, boosting curiosity. Preliminary support for this hypothesis comes from a recent study showing that the interest in a topic, as indexed by self-reported curiosity and interest ratings, was higher for participants who chose to watch a video from a set of options than for participants who watched a video without a choice of topic (Schutte \& Malouff, 2019). However, in this study, effects of choice were not controlled for effects of pre-choice preference. We conducted two experiments, in which we separately quantified effects of pre-choice preference and of choice itself, using an implicit (as well as an explicit) measure of curiosity. Furthermore, we manipulated two other factors that have been demonstrated to modulate curiosity, namely the expected value of the information and the size of the information update, to explore whether they mediate the effect of choice on curiosity.

Previous studies using similar lottery paradigms have already demonstrated that participants are more curious and/or willing to wait (or pay) more when the expected information is more positive (Charpentier et al., 2018; Kobayashi et al., 2019; van Lieshout et al., 2018), but also when the expected information update (i.e., uncertainty reduction) is greater (Kobayashi et al., 2019; van Lieshout et al., 2018). Accordingly, we considered that a potential boost of curiosity by choice might be mediated by an increase in either the expected value of the lottery, reflecting enhanced drive to maximize positive information ( $\mathrm{H} 1)$, and/or by the uncertainty of the lottery, reflecting enhanced drive to reduce the information gap and maximise the information update $(\mathrm{H} 2)$. The former hypothesis $(\mathrm{H} 1)$ posits that the mechanism of value enhancement underlying the boost of preference (Izuma et al., 2010; Sharot et al., 2009 ) is akin to that underlying the hypothesized boost of curiosity. The latter hypothesis $(\mathrm{H} 2)$ is grounded in the observation that when we have control over the environment it is more useful to 
maximise how much we learn about it. After all, it is more likely that the information we have gathered can be used to optimise future choices in such a controllable environment. Under this framework, the increase in curiosity for situations in which we have a choice should be largest under situations of largest uncertainty.

In both experiments, we manipulated the expected value of the lotteries and the amount of information to be gained from seeing their outcome (i.e., outcome uncertainty). If the effect of choice is a function of the subjective value of the lottery $(\mathrm{H} 1)$, then we should observe an interaction between choice and expected value. If the effect of choice varies with the strength of the drive to maximize the information update $(\mathrm{H} 2)$, then we should observe an interaction of choice and outcome uncertainty. Finally, we also considered a third hypothesis, according to which choice does not interact multiplicatively, but rather additively with the other driving factors of curiosity (H3). Under this hypothesis, we should observe a main effect of choice, in the absence of interactions with expected value or uncertainty. 


\section{Methods}

\section{Preregistration, data and code availability}

All data and code used for stimulus presentation and analysis are freely available on the Donders Repository (link will be available upon publication). The experiments and analyses were preregistered at the Open Science Framework (see preregistration: https://osf.io/gseqn).

\section{Participants}

We tested participants until we had 34 usable datasets for each experiment, in order to have $80 \%$ power of detecting at least a medium sized effect (Cohen's $d>0.5$ ) using a two-tailed T-test, alpha-level $p<0.05$, in a within-subject design.

Thirty-seven healthy individuals participated in Experiment 1 and another forty healthy individuals participated in Experiment 2. As preregistered, we excluded participants who missed more than $10 \%$ of total trials (i.e., criterion 1). Additionally, we excluded participants who scored poorly according to at least one of two other performance criteria. Criterion 2 was giving the same curiosity rating in more than $2 / 3$ of their responses in Experiment 1, or making the same willingness-to-wait decision in more than $90 \%$ of their responses in Experiment 2. Criterion 3 was ranking lower than 3 SDs below the mean in choice-preference coherence (i.e., frequency of trials on which a participant chose the same vase they had previously indicated to prefer). We excluded three participants from Experiment 1 (one based on criterion 2 and two based on criterion 3) and six participants from Experiment 2 (five based on criterion 2 and one based on criterion 3). No participants were excluded based on criterion 1 . The final sample in Experiment $1(n=34)$ included 26 women (age: $M=23.5, S D=4.5$ ); the final sample in Experiment 2 $(n=34)$ included 24 women (age: $M=23.5, S D=3.1$ ).

All participants had normal or corrected to normal vision. Both experiments were approved by the local ethics committee (CMO Arnhem-Nijmegen, The Netherlands) under the general ethics approval (CMO $2019 / 288$ v. 2.2). Prior to participation, all participants gave written informed consent according to the declaration of Helsinki.

\section{Procedure}

To assess the effect of choice on curiosity, we designed an experimental task in which we manipulated, on a trial-to-trial basis, whether participants made a choice or not and measured their subsequent curiosity. In each trial of the task, participants saw two lotteries, but only one was selected and played. Lotteries were depicted in the form of vases containing marbles, accompanied by a label indicating the amount of points associated with each marble. Each vase contained 20 marbles of two types: blue and red. A marble could be worth any number from 10 to 90 points, in steps of 10 (i.e., 10 points, 20, 30, etc.). The two types of marbles within a lottery always had different values. The distribution of marbles of each type within the vase ranged from 5 to $95 \%$ in steps of 5 (i.e., $5-95 \%$ vase, $10-90 \%, 15-85 \%$, etc.). On some trials, the participant chose the selected lottery, whereas on other trials the computer selected it for them. Participants were instructed that they would always earn a monetary payoff in proportion to the points associated with the outcome of the selected lottery, but that they could not always see the outcome after each trial. At the end of each trial, we assessed participants' curiosity using ratings (Experiment 1, explicit curiosity) or willingness-to-wait decisions (Experiment 2, implicit curiosity). 
The procedure for the two experiments was identical, with the key difference that in Experiment 2 participants could decide to see the outcome whereas in Experiment 1 this was randomly determined.

This resulted in three differences between the two experiments: 1) during the sign-up and instructions of Experiment 1 (explicit curiosity) the term "curiosity" was used to describe the task, whereas in Experiment 2 (implicit curiosity) "wanting to see the outcome" was used instead; 2 ) the final part of each trial differed between the two experiments (Figure 1); 3) this resulted in a slightly longer duration of Experiment 2 compared with Experiment 1 . We used willingness-to-wait to assess curiosity implicitly given that it is an acknowledged measure of the motivational value of an item (Frederick et al., 2002) and it has been used repeatedly to measure curiosity successfully (Kang et al., 2009; Marvin \& Shohamy, 2016; van Lieshout et al., 2018, 2019).

Before starting the experiment, participants received written instructions, after which they verbally summarised the task to the experimenter, and performed two practice blocks ( 20 trials in total). We instructed participants that each trial would begin with two lotteries of which only one would be selected (either by them or by the computer) and played. For each selected lottery, a marble would be picked at random based on the distribution in the vase, and they would earn the associated points. At the end of the experiment, all the points would be added and they would receive a bonus payment proportional to the final amount.

In both experiments (Figure 1), each trial began with a fixation cross shown for $0.5 \mathrm{~s}$, followed by a blank screen for $0.5 \mathrm{~s}$. Then, participants saw two lotteries side by side and had to indicate which lottery they preferred ("Vase 1 or Vase 2?"). Lotteries were presented until participants gave a preference response, up to a maximum of $10 \mathrm{~s}$. If no preference response was given within this time, the lotteries disappeared and participants had an additional $1 \mathrm{~s}$ interval to respond (i.e., adding up to a total response time of $11 \mathrm{~s})$. Participants then saw a blank screen for $0.5 \mathrm{~s}$, followed by a cue indicating whether they would choose themselves which of the two lotteries would be played ("Choose"; i.e., choice trials) or whether the lottery would be selected for them by the computer ("Match"; i.e., no-choice trials). This cue was presented for $1 \mathrm{~s}$ immediately followed by a response screen displaying the vase labels ("Vase 1" and "Vase 2") and a rectangle between them (in choice trials) or highlighting one of the labels (in no-choice trials). The position where each vase label was presented (i.e., left or right) was randomised on a trial-by-trial basis, so that participants had to attend to the screen instead of simply repeating the button press given at the preference stage of the trial. Participants always had to provide a response, either to choose or to match the option selected by the computer, within a maximum of $4 \mathrm{~s}$. Then, participants saw a blank screen for $0.5 \mathrm{~s}$, after which they saw the selected lottery again for $1 \mathrm{~s}$, followed by another blank screen for $0.5 \mathrm{~s}$. Up to this point trials were identical in both experiments.

In Experiment 1 (explicit curiosity, Fig. 1A), each trial continued with a screen displaying the prompt: "How curious are you about the outcome?", to which participants responded on a scale from 1 ("not curious") to 4 ("very curious") within a period of $4 \mathrm{~s}$. Participants responded with their right hand on a button box, using their index finger, middle finger, ring finger and little finger to indicate curiosity responses of $1,2,3$, and 4 , respectively. Following a $0.5 \mathrm{~s}$ blank screen, participants saw a final screen for $2 \mathrm{~s}$ that displayed the selected lottery and a box with the shown or hidden outcome. When the outcome was shown, the box contained a marble of the colour that had been picked (i.e., red or blue) and the points earned on that trial. When the outcome was hidden, the box contained a black marble and a string of question marks ("???"). The outcome box appeared with a $0.5 \mathrm{~s}$ delay after onset of to the lottery, and remained visible for $1.5 \mathrm{~s}$. The outcome was shown in half of the trials and hidden in the 
other half to ensure that the task remained engaging. This was randomly determined and did not depend on participants' curiosity ratings. We instructed participants that the points they earned on every trial would be added up to their final sum, regardless of whether they got to see the outcome or not.

In Experiment 2 (explicit curiosity, Fig. 1B), each trial continued with the prompt: "Do you want to see the outcome?", to which participants gave a binary "yes" or "no" response within a period of $4 \mathrm{~s}$. Participants responded with their right hand on a button box, using their index finger to indicate "yes" and their middle finger to indicate "no". If participants pressed "no", they continued to a final screen in which the outcome was hidden, immediately after a $0.5 \mathrm{~s}$ blank screen. If participants pressed "yes", they waited an additional 3 to $6 \mathrm{~s}$ before continuing to a final screen in which the outcome was shown. These displays were identical to those in Experiment 1. Critically, whether the outcome was shown or not was contingent on participants' willingness-to-wait responses. All participants were aware of these contingencies and of the additional delay, and knew that the points they earned on every trial would be added up to their final sum, regardless of whether they chose to see the outcome or not.

Trials could last a minimum and maximum of, respectively, 15 and $26.5 \mathrm{~s}$ in Experiment 1, and 15 and $32.5 \mathrm{~s}$ in Experiment 2. Throughout each trial, participants gave three responses: 1) to indicate which lottery they preferred, 2) to select a lottery (both for choice and no-choice trials), and 3) to indicate how curious they were (Experiment 1) or whether they wanted to wait to see the outcome (Experiment 2). If participants did not provide all of the three required responses within the allocated time, the trial was aborted and participants saw a warning message during the remaining duration of the trial reminding them to respond faster.

The experiment was divided into eight blocks, each followed by a break. Participants spent between $1 \mathrm{~h} 10 \mathrm{~min}$ and $1 \mathrm{~h} 40 \mathrm{~min}$ in the lab in Experiment 1, and $1 \mathrm{~h} 10 \mathrm{~min}$ and $1 \mathrm{~h} 50 \mathrm{~min}$ in Experiment 2. The duration of the session in Experiment 2 was affected by the number of trials in which participants chose to see the outcome. Prior to signing up to the experiment, participants were told they would be paid a fixed amount of 12 euro (Experiment 1) or 14 euro (Experiment 2) plus an undefined bonus. The base payment was proportional to the approximate duration of each experiment $(1 \mathrm{hr} 30 \mathrm{~min}$ and $1 \mathrm{hr} 45 \mathrm{~min}$, respectively). Based on the sum of the points participants earned during the task, they all received a bonus of 1.5 euro, resulting in a compensation of 13.50 euro (or 15.50 euro, if there was a delay) for Experiment 1 and of 15.50 euro (or 17.50 euro) for Experiment 2. The experiments were programmed and conducted using Presentation ${ }^{\circledR}$ software (Version 20.2, Build 07.25.18, Neurobehavioral Systems, Inc.).

Lotteries were paired creating 116 unique combinations (of 232 unique lotteries) that were presented twice: once for choice and once for no-choice trials. This resulted in a total of 232 trials, which appeared in random order. Even though each pair of lotteries was presented twice, vases were sampled from a list of 232 different configurations (i.e., marbles in different positions of the vase, even for vases of the same type), so each exact trial display was unlikely to repeat itself. Vase configurations were counterbalanced for colour so that neither red nor blue predominantly dominated the stimuli overall. 
A

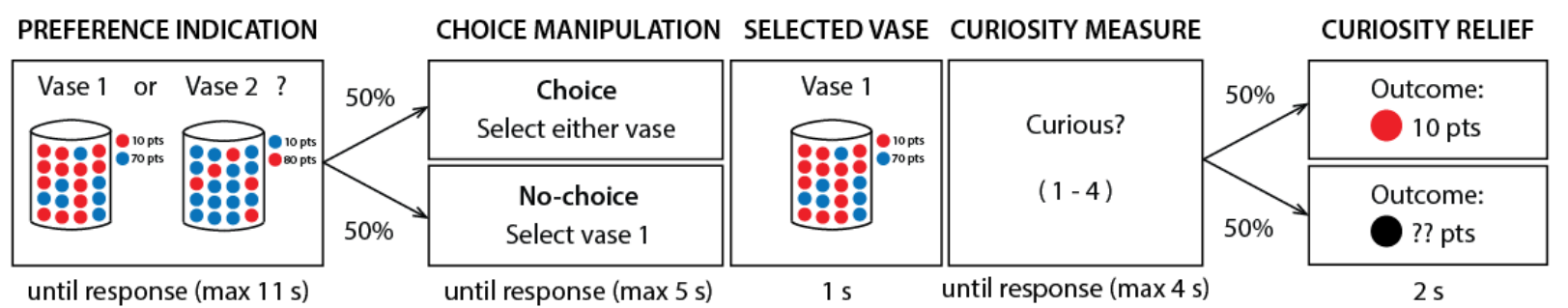

B

PREFERENCE INDICATION CHOICE MANIPULATION SELECTED VASE CURIOSITY MEASURE

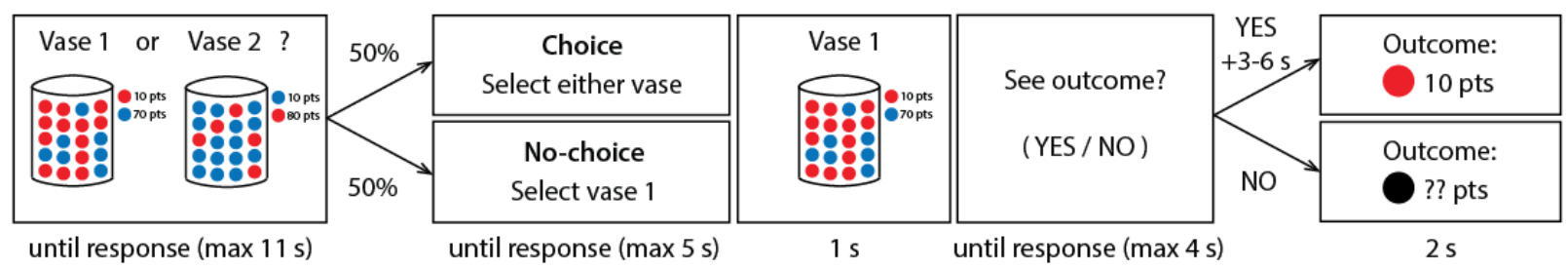

Figure 1. A. Trial schematic of Experiment 1 (explicit curiosity). At the beginning of each trial, participants saw two lotteries in the form of vases and indicated which one they preferred. Then, one of the two lotteries was selected: either by the participant (choice, $50 \%$ of trials), or by the computer (no-choice, $50 \%$ of trials). In no-choice trials, the computer selected the lottery initially preferred by the participant half of the time (no-choice-preferred, $25 \%$ of total trials), and the other lottery half of the time (no-choice-not-preferred, $25 \%$ of total trials). This distinction within no-choice trials was not made explicit to participants. After providing a response, participants saw the selected lottery. They then indicated how curious they were using a scale of 1 to 4 (not curious to very curious). Finally, participants saw a screen in which the outcome of the lottery was either shown (50\% of trials), or hidden $(50 \%$ of trials). Whether the outcome was shown or hidden was determined randomly, not contingent on participants' curiosity responses. Importantly, participants were awarded the points corresponding to the outcome of the lottery in every trial (added to their total point sum), regardless of whether they got to see the outcome or not. $\boldsymbol{B}$. Trial schematic of Experiment 2 (implicit curiosity). The task was identical to that of Experiment 1, up to (and including) when participants saw the selected lottery. After seeing the selected vase, participants had to indicate whether they wanted to see the outcome of the lottery or not. If participants wanted to see the outcome, they waited an additional 3 to $6 \mathrm{~s}$ before continuing to the final screen that showed the outcome. If they did not want to see the outcome, they continued without any additional delay to a final screen where the outcome was hidden. Importantly, as in Experiment 1, participants were awarded the points corresponding to the outcome of the lottery in every trial.

\section{Design}

To disentangle any effect of preference from the effect of choice, we ensured participants indicated their preferences at the beginning of each trial. Participants then received a choice trial half of the time $150 \%$ of trials) and a no-choice trial the other half ( $50 \%$ of trials). In choice trials, participants freely chose the lottery themselves. In no-choice trials, the computer selected participants' preferred lotteries half of the time ( $25 \%$ of trials) and their not-preferred lotteries the other half ( $25 \%$ of trials). We later sorted trials into three selection conditions: choice-preferred, no-choice-preferred, and no-choice-not-preferred trials. In no-choice-not-preferred trials, the computer had selected the lotteries participants had not 
preferred. By contrasting the choice-preferred with the no-choice-preferred selection condition we assessed the effect of choice independent of preference. By contrasting no-choice-preferred with no-choice-not-preferred we assessed the effect of preference under conditions of no choice.

Furthermore, we manipulated two other features of the lotteries: expected value and outcome uncertainty. We generated the values of expected value and outcome uncertainty by manipulating the distributions of marbles within a vase and their associated points.

The value of expected value for each lottery reflects its mean expected reward and was given by the average of the points associated with the marbles weighted by their distributions within the vase (i.e., probabilities of being picked). This was done as follows:

$$
\text { Expected value }(X)=\sum_{i=1}^{2} P\left(x_{i}\right) x_{i}
$$

where $P\left(x_{i}\right)$ denotes the probability that a marble type $i$ would be drawn, and $x_{i}$ denotes the points associated with such marble type (i.e., blue or red).

The value of outcome uncertainty for each lottery reflects the spread of the possible outcomes and was given by their variance. This was done as follows:

$$
\text { Outcome uncertainty }(X)=\sum_{i=1}^{2} P\left(x_{i}\right)\left(x_{i}-E V(X)\right)^{2}
$$

where $P\left(x_{i}\right)$ denotes the probability that a marble type $i$ would be drawn, and expected value $(X)$ denotes the expected value of the vase.

Note that we used a different calculation for outcome uncertainty than in a previous study using a similar task (van Lieshout et al., 2018) and different than we initially preregistered. Given that both metrics are nearly identical, and variance is a more common measure of uncertainty (Preuschoff et al., 2008; Symmonds et al., 2011), we decided to adopt this metric of outcome uncertainty.

Pairs of lotteries were closely matched in terms of both expected value and outcome uncertainty. By sampling a range of values of expected value and outcome uncertainty, we aimed to gain insight into the mechanisms underlying any effect of choice on curiosity. 


\section{Analysis}

\section{Pre-processing and filtering}

We read in, concatenated, filtered and summarised the data using RStudio (RStudio Team, 2020) with R 3.5.3 (R Core Team, 2019). We filtered out trials in which participants missed or did not respond within the allocated times for any of the three required responses $(0.37 \%$ of total trials in Experiment $1 ; 0.37 \%$ in Experiment 2), as well as choice trials in which participants had chosen a lottery other than their preferred one (2.73\% of total trials in Experiment 1; 2.93\% in Experiment 2). We sorted the remaining trials into three selection conditions: choice-preferred (48.61\% of included trials in Experiment 1; 48.42\% in Experiment 2), no-choice-preferred (25.72\% of included trials in Experiment 1; 25.79\% in Experiment 2), and no-choice-not-preferred trials (25.67\% of included trials in Experiment 1; $25.79 \%$ in Experiment 2).

\section{Mixed effects modelling using BRMS}

The dependent variable in Experiment 1 was curiosity rating, an ordinal variable containing values 1, 2, 3 and 4. In Experiment 2, the dependent variable was willingness-to-wait, a binary variable with values "no" and "yes" that we recoded to 0 and 1 , respectively, for this analysis.

The independent variables of interest were selection condition (choice-preferred, no-choice-preferred, and no-choice-not-preferred), expected value, and outcome uncertainty. Expected value and outcome uncertainty were continuous predictors, which we mean-centred and scaled. Selection condition was a factor with three levels, for which we created two sum-to-zero contrasts. We contrasted choice-preferred with no-choice-preferred trials to assess the effect of choice, and no-choice-preferred with no-choice-not-preferred trials to assess the effect of preference. With categorical predictors, BRMS provides estimates only for each contrast and not for the factor as a whole. Given that we were interested in disentangling the effects of choice and preference, this was suitable. Hence, in the results of the analysis with BRMS we report statistics only for each contrast and not for the factor of selection condition as a whole.

We modelled the data using the brm function of the brms package in $R$ (Bürkner, 2017, 2018), fitting a cumulative-logit model for the ordinal response data from Experiment 1, and a Bernoulli distribution model for the binomial response data from Experiment 2. We used the brms package instead of the initially planned approach (see preregistration) given that the brms package has functions for both ordinal and binomial data, allowing for a more consistent analysis approach. Furthermore, we performed additional statistical analyses using repeated measures ANOVA and its Bayesian equivalent (see Supplementary material), to verify the robustness of our results and to quantify the evidence for the null hypothesis for non-significant results.

Both models in brms (for Experiment 1 and Experiment 2, respectively) included predictors for the main effects of selection condition (represented as the two contrasts described earlier), expected value, and outcome uncertainty, as well as for the interactions between selection condition and expected value, and selection condition and outcome uncertainty, as fixed effects. The models included a full random effects structure (Barr, 2013; Barr et al., 2013), so that a random intercept and random slopes for all effects were included per participant. 
We fitted the models using four chains with 10,000 iterations (5,000 for warm up) and inspected the model summaries for convergence (paying attention to Rhat and effective sample size values), as well as the plots for chain iterations. We used the default priors of the brms package (Cauchy priors and LKJ priors for correlation parameters; Bürkner, 2017). We considered coefficients to be statistically significant if the associated $95 \%$ posterior credible intervals were non-overlapping with zero.

\section{Data visualisation}

For data visualisation, we recoded the values of the independent variable in Experiment 2 from "no" and "yes" to 0 and 100 , so that the mean implicit curiosity value reflected participants' percentage willingness-to-wait.

Furthermore, we transformed the dependent variables in both experiments (curiosity ratings in Experiment 1 and willingness-to-wait in Experiment 2) to avoid any effects of expected value in the outcome uncertainty plots and vice versa. Even though expected value and outcome uncertainty were manipulated independently and showed no significant linear correlation, these two predictors were operationalised in a way that high values of outcome uncertainty were only possible for trials with intermediate values of expected value. Plotting the raw data for each predictor without correcting for the other would distort its relationship with curiosity in the visualisation. To avoid this, we fitted a linear regression per participant and condition with curiosity as the dependent variable and outcome uncertainty as the predictor, and used the residuals plus the mean as the corrected curiosity values in the expected value visualisation. We performed the same procedure in the outcome uncertainty visualisation.

Next, we organised the data from the continuous predictors, expected value and outcome uncertainty, into four bins. We calculated the first, second, and third quartiles to be used as cut-offs between bins. We included trials with values below or equal to the first quartile in bin 1, trials above the first quartile and below or equal to the second quartile in bin 2, and so forth for bins 3 and 4 . Finally, we calculated the mean and the standard error (SEM) of the dependent variable for each bin and selection condition. We performed this separately for both expected value and outcome uncertainty. This visualisation is shown in Figure 2.

Additionally, to illustrate to what extent participants showed choice and preference effects, we calculated the mean curiosity ratings in Experiment 1 and percentage willingness-to-wait in Experiment 2 for each participant and selection condition. This visualisation is shown in Figure 3. 


\section{Results}

\section{Choice and preference independently enhanced curiosity}

Choice enhanced curiosity, such that participants were more curious about lotteries they had chosen themselves (curiosity ratings: $M=2.94, S D=0.33$; willingness to wait: $M=47.19 \%, S D=19.81 \%$ ) than about those that had been provided to them (matching their preference; curiosity ratings: $M=2.78$, $S D=0.3$; willingness to wait: $M=40.19 \%, S D=19.23 \%)$. This was the case when curiosity was measured explicitly (95\% Cl [0.46, 0.71]; Fig. 2A-B), as well as implicitly (95\% Cl [0.24, 0.64]; Fig. 2C-D). Additionally, we found an effect of preference, such that, when lotteries were provided to participants, they were more curious about lotteries they had indicated as preferred relative to their not-preferred ones (curiosity ratings: $M=2.44, S D=0.3$; willingness to wait: $M=30.66 \%, S D=18.63 \%$ ). Again, this was the case when curiosity was measured explicitly $(95 \% \mathrm{Cl}[0.57,0.85]$; Fig. $2 \mathrm{~A}-\mathrm{B})$, as well as implicitly (95\% $\mathrm{Cl}[0.24,0.77]$; Fig. 2C-D).
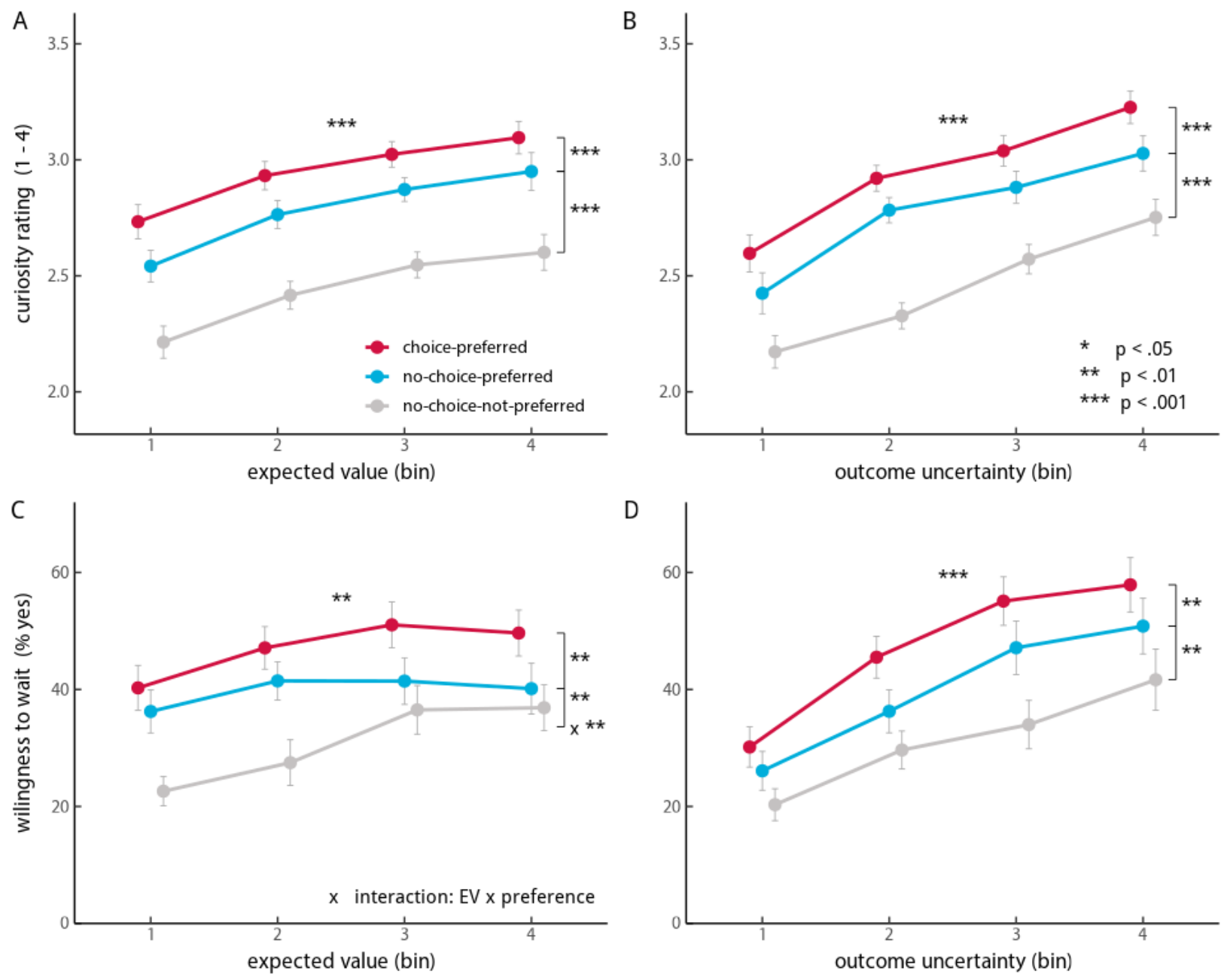

Figure 2. Choice (choice-preferred vs. no-choice-preferred conditions) and preference (no-choice-preferred vs. no-choice-not-preferred) enhanced curiosity measured both explicit $(\boldsymbol{A}, \boldsymbol{B})$ and implicitly $(\boldsymbol{C}, \boldsymbol{D})$. Curiosity ( $\boldsymbol{A}$ explicit, $\boldsymbol{C}$ implicit) increased monotonically as a function of outcome uncertainty. Curiosity ( $\boldsymbol{B}$ explicit, $\boldsymbol{D}$ implicit) increased monotonically as a function of expected value. Expected value, however, did not modulate implicit curiosity for preferred stimuli in the absence of choice ( $\boldsymbol{D}$, no-choice-preferred condition). Error bars represent the standard error of the mean (SEM). Significance levels plotted were obtained from the statistical analysis using repeated measures ANOVA. 
These effects of choice and preference on curiosity were present in a large proportion of the participants (Fig. 3).

\section{Curiosity increased as a function of expected value and outcome uncertainty}

Furthermore, we found that curiosity monotonically increased with expected value, such that participants were more curious the larger the reward they expected to earn. This was the case both
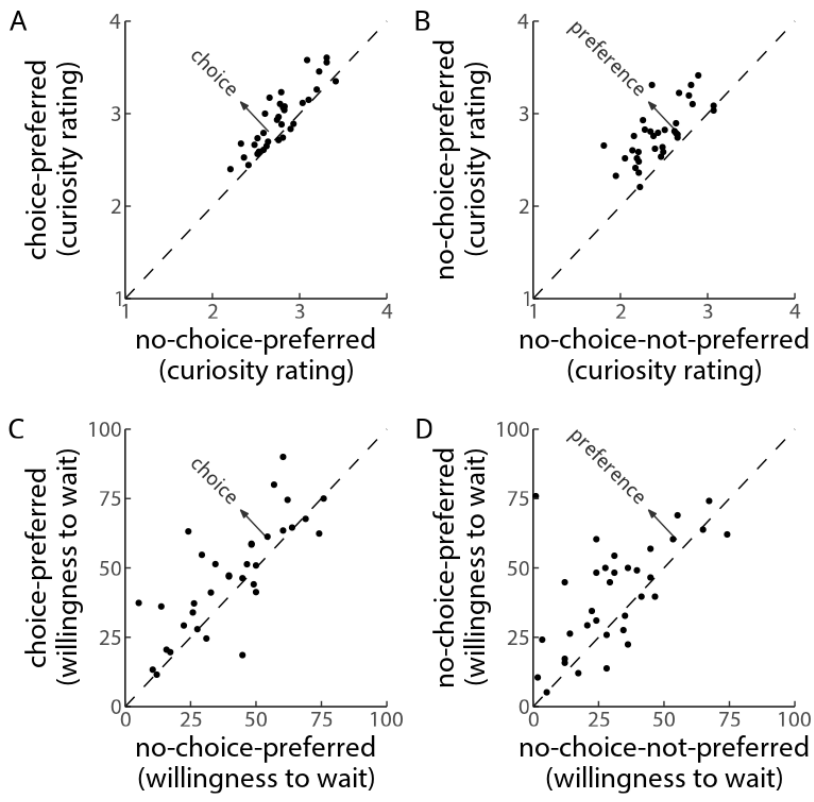

Figure 3. Most participants showed an effect of choice on curiosity, measured both explicit $(\boldsymbol{A})$ and implicitly $(\boldsymbol{C})$, as well as an effect of preference on curiosity ( $\boldsymbol{B}$ explicit, $\boldsymbol{D}$ implicit). Each point in the scatterplot represents one participant. The degree to which the point deviates from the diagonal in the 45-degree left-upwards direction (indicated by the arrow) represents the magnitude of each effect for a given participant. when curiosity was measured explicitly (95\% CI [0.24, 0.51]; Fig. 2A), as well as implicitly (95\% Cl [0.08, 0.31]; Fig. 2C).

Similarly, curiosity monotonically increased with outcome uncertainty, such that participants were more curious for more uncertain lotteries (i.e., the larger the information update they could gain). This was the case when curiosity was measured explicitly ( $95 \% \mathrm{Cl}[0.31,0.72]$; Fig. 2B), as well as implicitly (95\% Cl [0.24, 0.66]; Fig. 2D).

The effect of expected value on explicit curiosity did not differ between chosen and provided lotteries $(95 \% \mathrm{Cl}[-0.07,0.05])$, or between preferred and not-preferred lotteries $(95 \% \mathrm{Cl}[-0.10,0.11])$. While the effect of expected value on implicit curiosity did not differ between chosen and provided lotteries either $(95 \% \mathrm{Cl}[-0.09,0.07])$, it did differ significantly between preferred and not-preferred lotteries $(95 \% \mathrm{Cl}[-0.23,-0.03])$.

Additionally, the effect of outcome uncertainty on curiosity did not differ between selection conditions, whether curiosity was measured explicitly (choice vs. no-choice

$95 \% \mathrm{Cl}[-0.02,0.12] ;$ preferred vs. not-preferred $95 \% \mathrm{Cl}[-0.08,0.06])$ or implicitly (choice vs.

no-choice $95 \% \mathrm{Cl}[-0.07,0.11])$; preferred vs. not-preferred $95 \% \mathrm{Cl}[-0.07,0.17])$. 


\section{Discussion}

In the current set of studies, we assessed whether freely choosing which information to sample increased curiosity. We found that curiosity about the outcome of a lottery was enhanced for lotteries that were freely chosen, relative to lotteries that were equally preferred but not freely chosen. In the absence of choice, curiosity was higher for lotteries that were preferred than for those that were not preferred. These effects were consistent across participants. Furthermore, we investigated the effects of the expected value and the outcome uncertainty of the lottery. In line with previous findings (Charpentier et al., 2018; Kobayashi et al., 2019; van Lieshout et al., 2018), curiosity increased as a function of both these factors. However, the effect of choice on curiosity was independent of the expected value and outcome uncertainty of the lottery. Thus, choice boosted curiosity irrespective of how valuable the outcome was likely to be and irrespective of how much information could be gained from seeing the outcome.

Choice boosted curiosity both when participants reported their curiosity levels explicitly (Experiment 1) and when they did so implicitly, by deciding to "pay" with their time (i.e., willingness to wait) to see the outcome (Experiment 2). The fact that participants not only provided self-reports of being more curious, but were actually willing to give up a valuable resource (i.e., time) to see the outcome more frequently, makes it unlikely that this effect of choice on curiosity was due to participants complying with any inferred demand characteristics of the task.

Participants were more curious and more willing to wait after having made a choice. This was the case even though the subsequent curiosity relief by revealing the outcome did not serve an instrumental purpose (i.e., did not help them perform better in the task or maximise rewards in any way). Our finding generalizes the phenomenon of choice-induced preference change, observed in studies on value-based choice (Brehm, 1956; Izuma et al., 2010; Sharot et al., 2009, 2010), to the context of non-instrumental curiosity and information seeking. Thus, the value of information might be boosted by choice just as the value of an option is boosted by having chosen that option. Future studies might leverage pre- and post-choice neuro-economic preference tools to establish that choice indeed changes the subjective value that people assign to obtaining information. It is worth noting that in the current study we controlled for pre-choice preference by comparing those trials in which participants chose their preferred option with trials in which they did not have a choice but their preferred option was selected for them. Hence, our finding that choice boosted curiosity is not confounded by a pre-existing difference in preference between lotteries, but must reflect a choice-related change in the preference of information.

What psychological mechanism might underlie this choice-related boost in preference for information? A priori we considered several possibilities. First, choice might boost curiosity by enhancing our drive to maximize positive information. We obtain no evidence for an interaction between the effect of choice and the expected value of the lottery, so if choice boosts value, then it does so additively rather than multiplicatively. Second, choice might boost curiosity by enhancing our drive to minimize uncertainty. There was also no evidence for an interaction between the effect of choice and the outcome uncertainty of the lottery. Instead, we found that choice increased participants' curiosity at the same rate across all levels of expected value and outcome uncertainty. Thus, the effect of choice on curiosity was also additive, rather than multiplicative, with respect to the effect of outcome uncertainty. 
An intriguing question for future work pertains to the neural mechanism underlying the effect of the choice-related boost in preference for information. Given the link between midbrain dopamine neuronal firing and information prediction errors (Bromberg-Martin \& Hikosaka, 2011), it is plausible that the mechanism suggested to account for choice-induced preference change, involving feedback projections from the striatum to the midbrain (Cockburn et al., 2014), could also account for choice-induced increases in curiosity (or the value of information).

In addition, the noradrenaline system might play a modulatory role, given its implication in engagement or arousal, which is likely enhanced in conditions of increased autonomy (Howells et al., 2010; Sara \& Bouret, 2012; Varazzani et al., 2015). A role for noradrenaline in information seeking is also supported by recent evidence indicating that pharmacological blockade of noradrenergic receptor stimulation with propranolol decreased information gathering in a task requiring participants to uncover cards until they felt confident to make a guess about the predominant underlying colour (Hauser et al., 2018). Hence, the increased autonomy experienced in the choice trials of our task may have contributed to implicate the noradrenergic system as mentioned above.

In both experiments, we found that when lotteries were selected for participants without them having a choice, participants were more curious about lotteries that they preferred compared with those they did not. Preference enhanced curiosity and was, over all, independent of expected value and outcome uncertainty. This suggests that, preference for an option itself boosts the perceived value of information, hence increasing curiosity. Alternatively, it is possible that the mechanism underlying the effect of preference on curiosity was akin to that underlying the effect of choice, given that in our experiments participants had to provide a preference indication (i.e., engaging in a comparison process, making a decision, and a executing a response), which they might have experienced as a kind of choice.

Participants were more curious (Experiment 1) and willing to wait (Experiment 2) for lotteries with higher expected values. In other words, participants were more curious the more positive they expected the information to be. This concurs with previous findings showing that people prefer information about future desirable outcomes over information about undesirable outcomes (Charpentier et al., 2018; Kobayashi et al., 2019), and are even willing to pay for the positive information (Charpentier et al., 2018), perhaps reflecting a form of savouring (ligaya et al., 2016). This effect of expected value on curiosity was more marked than that observed in previous studies using a similar paradigm, but without the valuation and choice phases (van Lieshout et al., 2018, 2019, 2020). Unlike these previous studies, participants in the current study were explicitly instructed to make a value-based comparison between pairs of lotteries to indicate their preferred option and to subsequently choose one of them to be played. These aspects of the task likely increased the salience of the expected value of the lotteries. Other features of the present task might have further boosted participants' attention and/or engagement, including the longer duration of the lottery-presentation (up to $11 \mathrm{~s}$ in the current study versus $3 \mathrm{~s}$ in the previous studies), as well as the fact that in the present study participants got to see the outcome of their preferred lotteries in the majority of trials (75\%) whereas in the previous studies participants did not have a choice between lotteries.

Our findings are in line with preliminary evidence that autonomy boosts curiosity (Schutte \& Malouff, 2019), and go beyond that prior study by quantifying effects of choice and preference separately, and by demonstrating that these two factors boost curiosity independently. We also add to this prior study by showing that choice does not relate to expected value or outcome uncertainty, established factors 
known to play a role in curiosity. Finally, in addition to obtaining explicit curiosity ratings (similar to Schutte \& Malouff, 2019), we also measured curiosity in terms of more implicit willingness-to-wait decisions, well recognized to index the motivational value of an option, and less vulnerable to the impact of demand characteristics of a task than are explicit subjective ratings.

Curiosity (Gruber et al., 2014; Kang et al., 2009; Kidd \& Hayden, 2015) and choice (Cockburn et al., 2014; Murty et al., 2015) have both been shown to boost learning and memory, and these effects have both implicated coupling between reward-related areas (i.e., ventral striatum) and the hippocampus. However, to our knowledge, the exact nature of the link between curiosity and choice in boosting memory remains unknown. Our findings raise the hypothesis that a choice-induced curiosity boost might contribute to a choice-induced memory boost and might be one of the first steps in bridging the gap between the choice and curiosity frameworks, for instance, in terms of their consequences for learning and memory (Cockburn et al., 2014; Gruber et al., 2014; Kang et al., 2009; Kidd \& Hayden, 2015; Murty et al., 2015). The current paradigm may be leveraged in future work aimed at linking task engagement and motivation with curiosity and dopamine or noradrenaline, as well as investigating further into the nature of the relationship between choice, curiosity, learning and memory.

Given the abundance of sources of information in our technological era, it is key to understand how selecting between them piques our curiosity. Our findings suggest that choice has a key role in determining what information we are more likely to consume. Free choice and autonomy, as tools to enhance curiosity and boost learning and memory, open up promising avenues for future research and applications. One clear domain of application is education, where the benefits of autonomy and curiosity for learning have been long recognised, but remain to be exploited (Borg \& Al-Busaidi, 2012; Yen Dwee \& Anthony, 2017). Advancing our understanding of the benefits of choice on curiosity and learning will contribute to the tailoring of more robust educational interventions/programmes.

In sum, our study shows that when we make an autonomous/free choice regarding what source to sample information from, we become more curious about that information. This boost in curiosity is independent of the promise of that information for either maximizing positive beliefs or reducing uncertainty. Furthermore, this effect of choice on curiosity can be seen both with explicit reports of curiosity as well as with implicit behavioural measures, such as being willing to wait for information. 


\section{References}

Ariely, D., \& Norton, M. I. (2008). How actions create - not just reveal - preferences. Trends in Cognitive Sciences, 12(1), 13-16. https://doi.org/10.1016/j.tics.2007.10.008

Barr, D. J. (2013). Random effects structure for testing interactions in linear mixed-effects models.

Frontiers in Psychology, 4. https://doi.org/10.3389/fpsyg.2013.00328

Barr, D. J., Levy, R., Scheepers, C., \& Tily, H. J. (2013). Random effects structure for confirmatory hypothesis testing: Keep it maximal. Journal of Memory and Language, 68(3). https://doi.org/10.1016/j.jml.2012.11.001

Bennett, D., Bode, S., Brydevall, M., Warren, H., \& Murawski, C. (2016). Intrinsic Valuation of Information in Decision Making under Uncertainty. PLOS Computational Biology, 12(7), e1005020. https://doi.org/10.1371/journal.pcbi.1005020

Blanchard, T. C., Hayden, B. Y., \& Bromberg-Martin, E. S. (2015). Orbitofrontal Cortex Uses Distinct Codes for Different Choice Attributes in Decisions Motivated by Curiosity. Neuron, 85(3), 602-614. https://doi.org/10.1016/j.neuron.2014.12.050

Borg, S., \& Al-Busaidi, S. (2012). Teachers' beliefs and practices regarding learner autonomy. ELT Journal, 66(3), 283-292. https://doi.org/10.1093/elt/ccr065

Brehm, J. W. (1956). Postdecision changes in the desirability of alternatives. The Journal of Abnormal and Social Psychology, 52(3), 384-389. https://doi.org/10.1037/h0041006

Bromberg-Martin, E. S., \& Hikosaka, O. (2011). Lateral habenula neurons signal errors in the prediction of reward information. Nature Neuroscience, 14(9), 1209-1216. https://doi.org/10.1038/nn.2902

Bürkner, P.-C. (2017). brms: An R Package for Bayesian Multilevel Models Using Stan. Journal of Statistical Software, 80(1). https://doi.org/10.18637/jss.v080.i01

Bürkner, P.-C. (2018). Advanced Bayesian Multilevel Modeling with the R Package brms. The R Journal, 10(1), 395. https://doi.org/10.32614/RJ-2018-017 
Charpentier, C. J., Bromberg-Martin, E. S., \& Sharot, T. (2018). Valuation of knowledge and ignorance in mesolimbic reward circuitry. Proceedings of the National Academy of Sciences, 115(31), E7255E7264. https://doi.org/10.1073/pnas.1800547115

Cockburn, J., Collins, A. G. E., \& Frank, M. J. (2014). A Reinforcement Learning Mechanism Responsible for the Valuation of Free Choice. Neuron, 83(3), 551-557. https://doi.org/10.1016/j.neuron.2014.06.035

Frederick, S., Loewenstein, G., \& O’Donoghue, T. (2002). Time Discounting and Time Preference: A Critical Review. Journal of Economic Literature, 40(2), 351-401. https://doi.org/10.1257/002205102320161311

Gruber, M. J., Gelman, B. D., \& Ranganath, C. (2014). States of Curiosity Modulate HippocampusDependent Learning via the Dopaminergic Circuit. Neuron, 84(2), 486-496. https://doi.org/10.1016/j.neuron.2014.08.060

Hauser, T. U., Moutoussis, M., Purg, N., Dayan, P., \& Dolan, R. J. (2018). Beta-Blocker Propranolol Modulates Decision Urgency During Sequential Information Gathering. The Journal of Neuroscience, 38(32), 7170-7178. https://doi.org/10.1523/JNEUROSCI.0192-18.2018 Howells, F. M., Stein, D. J., \& Russell, V. A. (2010). Perceived mental effort correlates with changes in tonic arousal during attentional tasks. 15.

ligaya, K., Story, G. W., Kurth-Nelson, Z., Dolan, R. J., \& Dayan, P. (2016). The modulation of savouring by prediction error and its effects on choice. Elife, 5, e13747.

Izuma, K., Matsumoto, M., Murayama, K., Samejima, K., Sadato, N., \& Matsumoto, K. (2010). Neural correlates of cognitive dissonance and choice-induced preference change. Proceedings of the National Academy of Sciences, 107(51), 22014-22019. https://doi.org/10.1073/pnas.1011879108 JASP (Version 0.13). (2020). [Computer software]. JASP Team. 
Kang, M. J., Hsu, M., Krajbich, I. M., Loewenstein, G., McClure, S. M., Wang, J. T., \& Camerer, C. F. (2009). The Wick in the Candle of Learning: Epistemic Curiosity Activates Reward Circuitry and Enhances Memory. Psychological Science, 20(8), 963-973. https://doi.org/10.1111/j.14679280.2009.02402.x

Kidd, C., \& Hayden, B. Y. (2015). The Psychology and Neuroscience of Curiosity. Neuron, 88(3), 449-460. https://doi.org/10.1016/j.neuron.2015.09.010

Kobayashi, K., Ravaioli, S., Baranès, A., Woodford, M., \& Gottlieb, J. (2019). Diverse motives for human curiosity. Nature Human Behaviour. https://doi.org/10.1038/s41562-019-0589-3

Marvin, C. B., \& Shohamy, D. (2016). Curiosity and reward: Valence predicts choice and information prediction errors enhance learning. Journal of Experimental Psychology: General, 145(3), 266272. https://doi.org/10.1037/xge0000140

Murty, V. P., DuBrow, S., \& Davachi, L. (2015). The Simple Act of Choosing Influences Declarative Memory. The Journal of Neuroscience, 35(16), 6255-6264. https://doi.org/10.1523/JNEUROSCI.4181-14.2015

Preuschoff, K., Quartz, S. R., \& Bossaerts, P. (2008). Human Insula Activation Reflects Risk Prediction Errors As Well As Risk. Journal of Neuroscience, 28(11), 2745-2752. https://doi.org/10.1523/JNEUROSCl.4286-07.2008

R Core Team. (2019). R: A language and environment for statistical computing. R Foundation for Statistical Computing, Vienna, Austria. https://www.R-project.org/

Rodriguez Cabrero, J. A. M., Zhu, J.-Q., \& Ludvig, E. A. (2019). Costly curiosity: People pay a price to resolve an uncertain gamble early. Behavioural Processes, 160, 20-25. https://doi.org/10.1016/j.beproc.2018.12.015

RStudio Team. (2020). RStudio: Integrated Development for R. RStudio, PBC, Boston, MA. http://www.rstudio.com/ 
Sara, S. J., \& Bouret, S. (2012). Orienting and Reorienting: The Locus Coeruleus Mediates Cognition through Arousal. Neuron, 76(1), 130-141. https://doi.org/10.1016/j.neuron.2012.09.011

Schutte, N. S., \& Malouff, J. M. (2019). Increasing curiosity through autonomy of choice. Motivation and Emotion, 43(4), 563-570. https://doi.org/10.1007/s11031-019-09758-w

Sharot, T., De Martino, B., \& Dolan, R. J. (2009). How Choice Reveals and Shapes Expected Hedonic Outcome. Journal of Neuroscience, 29(12), 3760-3765. https://doi.org/10.1523/JNEUROSCI.4972-08.2009

Sharot, T., \& Sunstein, C. R. (2020). How people decide what they want to know. Nature Human Behaviour, 4(1), 14-19. https://doi.org/10.1038/s41562-019-0793-1

Sharot, T., Velasquez, C. M., \& Dolan, R. J. (2010). Do Decisions Shape Preference?: Evidence From Blind Choice. Psychological Science, 21(9), 1231-1235. https://doi.org/10.1177/0956797610379235

Symmonds, M., Wright, N. D., Bach, D. R., \& Dolan, R. J. (2011). Deconstructing risk: Separable encoding of variance and skewness in the brain. Neurolmage, 58(4), 1139-1149. https://doi.org/10.1016/j.neuroimage.2011.06.087

van Lieshout, L. L. F., de Lange, F., \& Cools, R. (2020). Curiosity: An appetitive or an aversive drive? [Preprint]. PsyArXiv. https://doi.org/10.31234/osf.io/s3zp4

van Lieshout, L. L. F., Traast, I., de Lange, F. P., \& Cools, R. (2019). Curiosity or savouring? Information seeking is modulated by both uncertainty and valence. [Preprint]. PsyArXiv. https://doi.org/10.31234/osf.io/5y6pz

van Lieshout, L. L. F., Vandenbroucke, A. R. E., Müller, N. C. J., Cools, R., \& de Lange, F. P. (2018). Induction and relief of curiosity elicit parietal and frontal activity. The Journal of Neuroscience, 2816-2817. https://doi.org/10.1523/JNEUROSCI.2816-17.2018 
Varazzani, C., San-Galli, A., Gilardeau, S., \& Bouret, S. (2015). Noradrenaline and Dopamine Neurons in the Reward/Effort Trade-Off: A Direct Electrophysiological Comparison in Behaving Monkeys. Journal of Neuroscience, 35(20), 7866-7877. https://doi.org/10.1523/JNEUROSCI.0454-15.2015

Yen Dwee, C., \& Anthony, E. M. (2017). Learner Autonomy in University English Classrooms: Teachers' Perceptions and Practices. International Journal of Applied Linguistics and English Literature, 6(2), 19. https://doi.org/10.7575/aiac.ijalel.v.6n.2p.19 


\section{Supplementary material}

\section{Analysis}

\section{Repeated measures ANOVA}

We performed additional statistical analysis using repeated measures ANOVA. By applying two alternative statistical analyses (brms and ANOVA) with coinciding results we were able to verify the robustness of our results and demonstrate that our conclusions do not depend on the analytical framework employed.

For this analysis, we transformed and summarised the data in RStudio (RStudio Team, 2020), adjusted it to wide format and ran repeated measures ANOVAs in SPSS. We transformed the continuous predictors, expected value and outcome uncertainty, into factors with two levels: low and high. We used the median as the cut-off between levels, including trials with values equal to the median into the category with the lesser amount of data (low level for expected value, and high level for outcome uncertainty). We performed this transformation separately for both expected value (median $=50$ ) and outcome uncertainty (median $=222.75$ ).

We ran $3 \times 2 \times 2$ repeated measures ANOVAs with within-subject factors: selection condition (choice-preferred, no-choice-preferred, no-choice-not-preferred), expected value (low, high), and outcome uncertainty (low, high). The dependent variable was mean curiosity rating in Experiment 1 (between 1 and 4), and proportion willingness-to-wait in Experiment 2 (from 0 to 1, given that "no" was coded as 0 and "yes" as 1). We report the significance estimates corrected against violations of sphericity using Greenhouse-Geisser.

When observing a significant main effect of or an interaction with selection condition, we ran two follow-up repeated measures ANOVAs to assess whether this significant result was due to an effect of choice, preference, or both. To this end, we included only choice-preferred and no-choice-preferred trials to assess the effect of choice, and only no-choice-preferred and no-choice-not-preferred trials to assess the effect of preference, with all other variables identical to the full model described earlier.

\section{Bayesian Repeated Measures ANOVAs in JASP}

We performed the Bayesian equivalent of the repeated measures ANOVAs described above, in JASP $(J A S P, 2020)$. This allowed us to quantify the evidence for the null hypothesis in those cases in which the frequentist repeated measures ANOVA did not yield a significant result. We used the default Cauchy prior to compute Bayes Factors (BF) for each effect. We used model averaging across matched models to get a single BF for each effect in the repeated measures ANOVA. This BF reflects the change from prior to posterior inclusion odds. It can intuitively be understood as the amount of evidence that the data gives for including an experimental factor in a model. The BF will converge to zero when the factor should not be included in the model, or to infinity when the factor should be included in the model. Values close to one indicate that there is not enough evidence for either conclusion.

\section{Results}

Choice enhanced curiosity both when curiosity was measured explicitly $\left(F_{(1,33)}=27.43, p=9.156 \mathrm{e}^{-6}\right.$,

$\eta_{\mathrm{p}}{ }^{2}=.454, B F=1072.388$; RM with 3-level condition factor: $F_{(1.616,53.339)}=87.896, p=4.4586 \mathrm{e}^{-16}$, $\left.\eta_{p}^{2}=.727, B F=2.779 \mathrm{e}^{30}\right)$, as well as implicitly $\left(F_{(1,33)}=9.556, p=.004, \eta_{p}^{2}=.225, B F=34.168\right.$; RM with 
3-level condition factor: $\left.F_{(1.568,51.757)}=14.807, p=3.612 \mathrm{e}^{-5}, \eta_{\mathrm{p}}{ }^{2}=.31, B F=1.54 \mathrm{e}^{9}\right)$. Additionally, when lotteries were provided to participants, preference enhanced curiosity both measured explicitly $\left(F_{(1,33)}=82.006, p=1.8262 \mathrm{e}^{-10}, \eta_{\mathrm{p}}{ }^{2}=.713, B F=1.585 \mathrm{e}^{13}\right)$ and implicitly $\left(F_{(1,33)}=9.958, p=.003, \eta_{\mathrm{p}}{ }^{2}=.232\right.$, $B F=650.177)$.

Furthermore, curiosity monotonically increased with expected value, both when it was measured explicitly $\left(F_{(1,33)}=29.615, p=5.005 \mathrm{e}^{-6}, \eta_{\mathrm{p}}{ }^{2}=.473, B F=9.111 \mathrm{e}^{9}\right)$, as well as implicitly $\left(F_{(1,33)}=10.576\right.$, $\left.p=.003, \eta_{\mathrm{p}}{ }^{2}=.243, B F=57.979\right)$. Similarly, curiosity monotonically increased with outcome uncertainty, both when measured explicitly $\left(F_{(1,33)}=33.35, p=1.872 \mathrm{e}^{-6}, \eta_{\mathrm{p}}{ }^{2}=.503, B F=2.907 \mathrm{e}^{24}\right)$, as well as implicitly $\left(F_{(1,33)}=31.54, p=2.992 \mathrm{e}^{-6}, \eta_{\mathrm{p}}^{2}=.489, B F=6.499 \mathrm{e}^{16}\right)$.

The effect of expected value on explicit curiosity did not differ between chosen and provided lotteries or between preferred and not-preferred lotteries (RM with 3-level condition factor: $F_{(1.6,52.816)}=0.352$, $\left.p=.658, \eta_{p}^{2}=.011, B F=0.058\right)$. While the effect of expected value on implicit curiosity did not differ between chosen and provided lotteries $\left(F_{(1,33)}=2.889, p=.099, \eta_{p}{ }^{2}=.08, B F=0.401\right)$, whereas it did differ significantly between preferred and not-preferred lotteries $\left(F_{(133)}=10.041, p=.003, \eta_{p}^{2}=.233\right.$, $B F=3.465 ; \mathrm{RM}$ with a 3-level condition factor: $\left.F_{(1.892,62.429)}=6.211, p=.004, \eta_{\mathrm{p}}^{2}=.158, B F=0.685\right)$. The effect of outcome uncertainty on curiosity did not differ between selection conditions, whether curiosity was measured explicitly (RM with 3-level condition factor: $F_{(1.926,63.574)}=1.567, p=.217, \eta_{\mathrm{p}}^{2}=.045$, $B F=0.082)$ or implicitly (RM with 3-level condition factor: $F_{(1.809,59.690)}=2.128, p=.133, \eta_{\mathrm{p}}{ }^{2}=.061$, $B F=0.112$ ). 